\title{
IGEL PHANTOM : AN INNOVATIVE MODEL FOR USG GUIDED NEEDLING TRAINING
}

Jesto Kurian*, Sreechith P Karippuram, Isaac Babu, Sachin George

Rajagiri Hospital, Aluva, Kochi, India

IGEL Phantom display \& Sonoimage

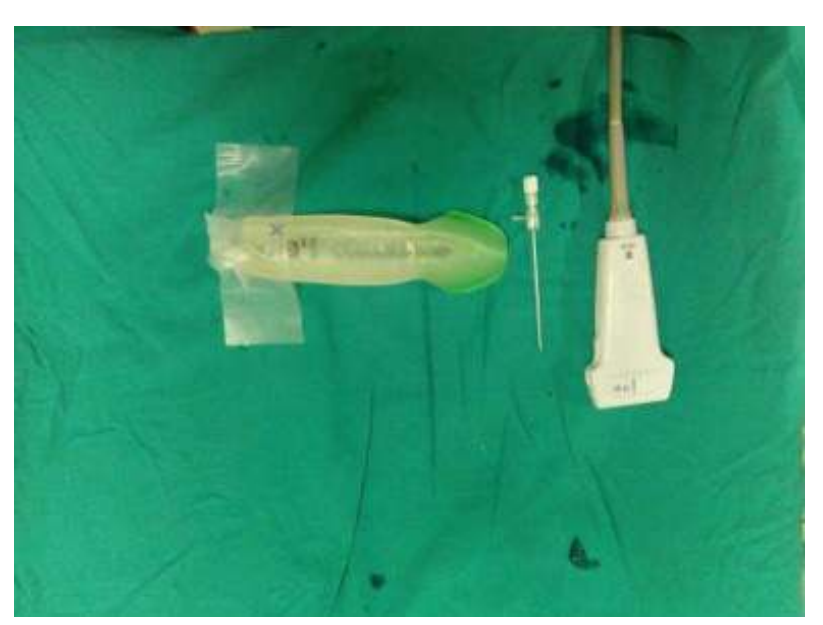

Figure 1

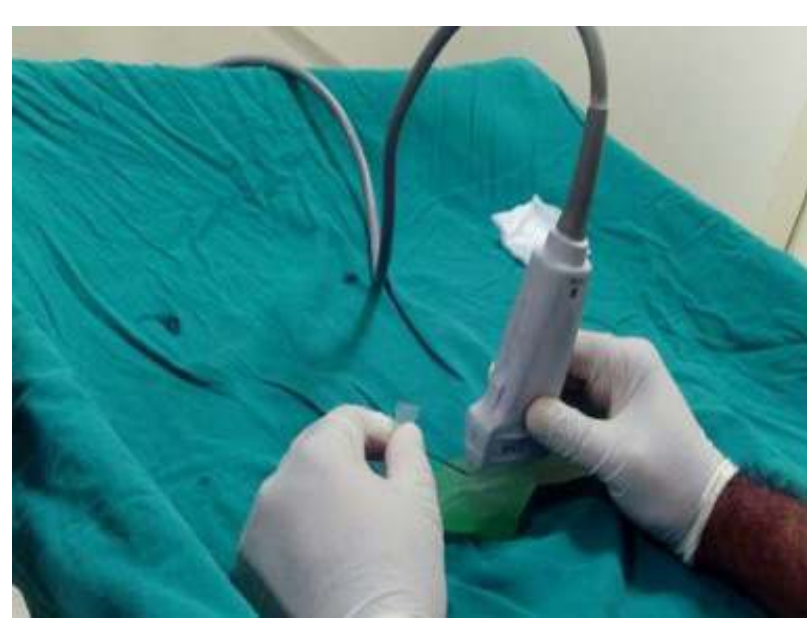

Figure 2

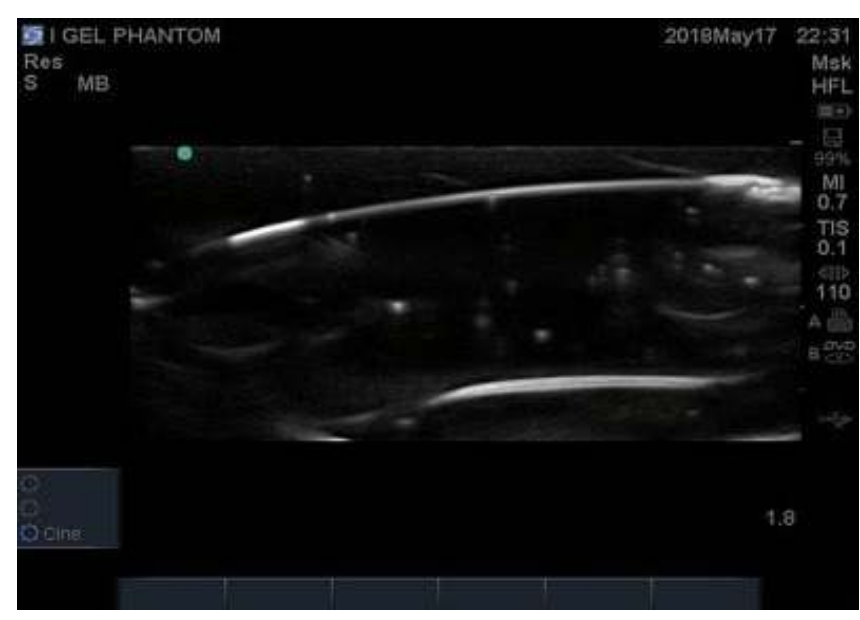

Figure 3

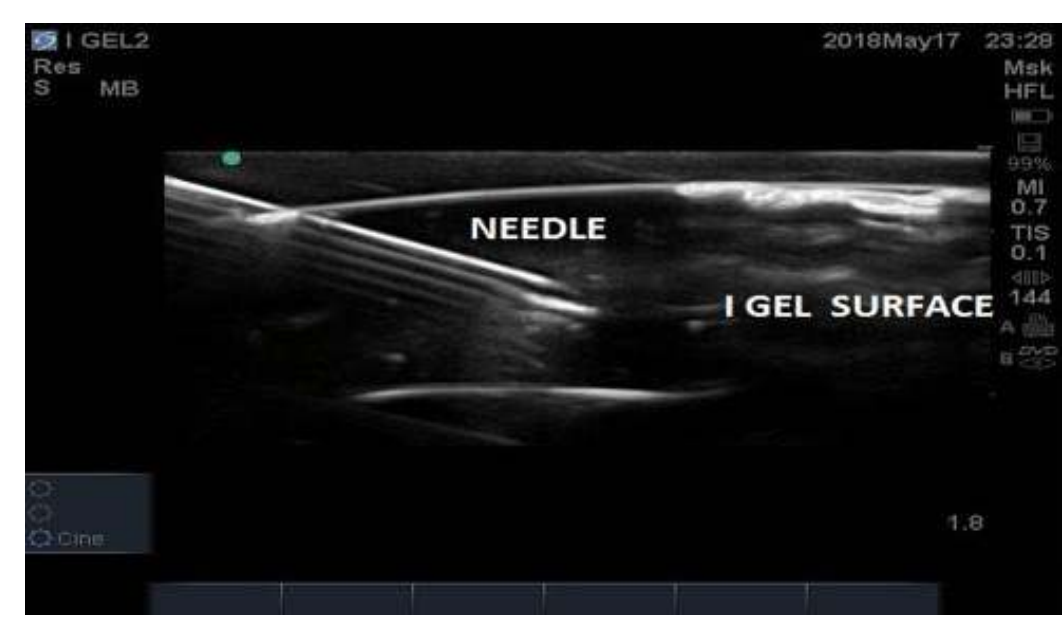

Figure 4

\section{Introduction}

Attaining skill in USG guided regional anesthesia technique takes a long learning curve .The availability of cost effective training phantoms is a major hindrance.

Igel is a second generation supraglottic device developed by Dr. Muhammed. The use of Igel as a training phantom has never been explained earlier. We report the use of Igel as an innovative method and training phantom for needling under USG guidance.

\section{Materials and method}

No.4 / N0.5 Igel

Transparent jelly

$16 \mathrm{G}$ intracath needle

USG machine with linear probe $(5-13 \mathrm{MHz})$

Igel is placed over a flat surface and the hollow of the I gel is filled with transparent jelly and both side of I gel is close during a adhesive tape(fig1). This will create a hyperechoic linear artefact which simulates the target on the sonoimage(fig2). The usg probe is placed longitudinaly along the long axis of Igel just proximal to the non inflatable cuff (fig3). The $16 \mathrm{G}$ needle is placed near the usg probe at around 30 degree angle to the surface of Igel and try to pierce the I gel and the needle will pass smoothly through the igel surface. You will find the entire length of needle passing through the igel and a linear hyperechoic line will be seen as the target(fig4). This will create a real time simulation of parenchymal tissue. Needling can be repeated several times without damaging the Igel.

\section{References}

\section{Discussion}

The advances in ultrasound technology like 3D and 4D USG and in needles like echogenic needles has increased the interest in regional anaesthesia techniques specially USG guided peripheral nerve blocks(5). But for acquiring proper skill set in these technique it takes a long learning curve. The major hindrance in this regard is the non-availability of proper training tools

The commercially available training tools are namely phantoms and simulation videos. The training phantom include blue phantom, gelatin based phantom and agar based phantom. The most popular among these is the blue phantom. But it is expensive and not freely available. The other phantoms like gelatin based phantom and agar based phantom lack shelf life and is time consuming to prepare $(1,2,3)$

We tried I gel as an innovative tool as a training phantom for needle manipulation under USG guidance.

\section{Outcome}

The sono image quality is comparable with other available phantom models. The image also simulates real time body tissue.

The advantages of Igel phantom are: easy availability, durability, cost effectiveness and shelf life.

\section{Conclusion}

Igel phantom can be a cost effective and durable training phantom for learning needle manipulation under USG guidance specially for novice interest in USG guided peripheral nerve blocks. With further studies and modifications Igel phantom can be a go to training tool for learning the skill of needle manipulation under USG guidance for regional anaesthesiologists.

1. Fredfeldt KE: An easily made ultrasound biopsy phantom. J Ultrasound Med 5:295

2. Silver B, Metzger TS, Matalon TA: A simple phantom for learning needle placement for sonographically guided biopsy. AJR 1542347

Hocking G, Hebard S, Mitchell CH. A review of the benefits and pitfalls of phantoms in ultrasound-guided regional anesthesia. Reg Anesth Pain Med 2011; 36:162-170.

Nelson, T. R. \& Pretorius, D. H. 1992 Three-dimensional ultrasound of fetal surface features. Ultrasound Obstet. Gynecol. 2, 166- 174

Deam RK, Kluger R, Barrington MJ, McCutcheon CA. Investigation of a new echogenic needle for use with ultrasound peripheral nerve blocks. Anaesthesia and Intensive Care 2007; 35: 582-6.
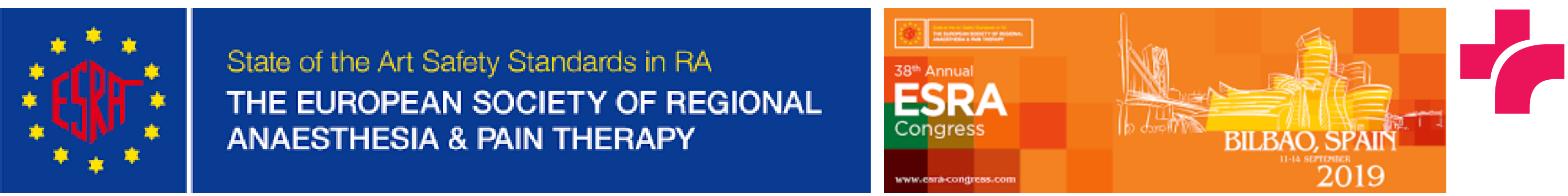